\title{
Calculating Reactive Power Compensation for Large-Scale Street Lighting
}

Sebastian Ernst ${ }^{1}$, Leszek Kotulski ${ }^{1}$, Tomasz Lerch ${ }^{2}$, Michał Rad ${ }^{2}$, Adam Sȩdziwy ${ }^{1}$, and Igor Wojnicki ${ }^{1(凶)}$

1 Department of Applied Computer Science, AGH University of Science and Technology, al. Mickiewicza 30, 30-059 Kraków, Poland

wojnicki@agh.edu.pl

2 Department of Power Electronics and Energy Control Systems, AGH University of Science and Technology, al. Mickiewicza 30, 30-059 Kraków, Poland

\begin{abstract}
LED-based street lighting installations generate reactive power, particularly when they are dynamically dimmed. It contributes to power loss and efficiency reduction of the grid. The reactive power can be compensated by installing additional dynamically connected inductors in lighting control cabinets. However such an approach significantly increases the cost of the lighting infrastructure. The goal of this paper is to propose another, low cost approach to reactive power compensation for dynamically dimmed lighting installations. It is based on connecting fixed settings inductors at lighting control cabinets. The inductors settings are calculated by the proposed algorithm for city-scale lighting systems. Its objective is to completely eliminate capacitive reactive power and to keep inductive reactive power within acceptable limits.
\end{abstract}

Keywords: Street lighting $\cdot$ Reactive power $\cdot$ LED lighting $\cdot$ Reactive power compensation

\section{Introduction}

In the last years we are witnessing a dynamic growth of usage of the solid state lighting technology. This is caused and stimulated by several factors, such as the demand of energy saving; physical properties of solid state light sources: neglectable onset time, dimming capabilities, high expected lifespan; continuously dropping prices of LED fixtures.

The annual global energy usage related to outdoor lighting is assessed to have $12-15 \%$ share in the total energy consumption [10]. In this context even a small improvement in lighting energy efficiency, e.g., of the order of $1 \%$, yields significant total savings, due to the effect of scale. The above reasoning has caused development of a broad scope of methods of power usage reduction [12] and sustainable maintenance of public lighting, beginning from the well suited 
lighting projects [6,9] relying on GIS-based inventory data [20,21], application of control systems [5,18,22], to sophisticated methods of tunnel illumination $[16,17]$ or improving reflective properties of road surface [19].

One of the basic benefits of retrofitting lighting installations with LED sources is a radical drop of energy usage of the order up to $60 \%[1,25]$. Additional savings can be achieved by adjusting luminous fluxes of luminaires when road and environment conditions change [24]. However, while dimming a luminaire to adjust lighting levels to particular needs saves energy, there is a side effect. It is a growth of capacitive reactive power in the power network, expressed interchangeably by means of the trigonometric values $\tan \varphi$ and $\cos \varphi$ known as power factor. As long as the power driver does not operate at its full capacity it dims the luminaire and introduces reactive power.

Reactive power is charged for both electric energy producers and consumers. A detailed insight into tariffs and pricing options for energy producers but also transmission providers can be found in [8]. In the case of customers it also depends on a regulatory framework [15].

Reactive power can be compensated by additional hardware components either attached to particular lamps [3,13] or introduced to a power grid. Although compensation as such is an easy task it becomes nontrivial in the context of a large (i.e., containing tens of thousands of light points), dynamically dimmed, LED-based lighting installation, powered by multiple, independent control cabinets, where the reactive power level is not constant but changes in an unpredictable manner.

The main goal of this paper is to introduce an algorithm that provides settings for static reactive power compensation for street lighting with adaptive control at each of the lighting control cabinets.

\subsection{Capacitive Reactive Power Compensation}

Let $u(t)$ and $i(t)$ denote respectively the voltage and current in an alternating current electric circuit, at a given time $t$. Moreover let us assume that both waveforms are sinusoids with the period $T$ (hence the angular frequency for both is $\left.\omega=\frac{2 \pi}{T}\right)$. A presence of a capacitor and/or an inductive coil in a circuit may cause a phenomenon of a phase shift between current and voltage which manifests as an additional term $\varphi$ in the waveform $i(t)=i_{0} \sin (\omega t+\varphi)$ (or $\left.u(t)=u_{0} \sin (\omega t-\varphi)\right)$.

An RMS (Root-Mean-Squared) voltage value is calculated according to the following definition:

$$
U=\sqrt{\frac{1}{T} \int_{0}^{T} u^{2}(t) d t .}
$$

An RMS current value is given by

$$
I=\sqrt{\frac{1}{T} \int_{0}^{T} i^{2}(t) d t .}
$$


To obtain an active power we compute the integral

$$
P=\frac{1}{T} \int_{0}^{T} u(t) i(t) d t
$$

In turn, an apparent power is given by

$$
S=U I
$$

For sinusoidal waveforms of voltage and current, the power equation is met:

$$
S^{2}=P^{2}+Q^{2}
$$

Using the Eq. 5 one can obtain straightforwardly a reactive power:

$$
Q^{2}=\sqrt{S^{2}-P^{2}}
$$

The power factor $(\cos \varphi)$ and $\tan \varphi$ values are calculated as

$$
\cos \varphi=\frac{P}{S}, \quad \tan \varphi=\frac{Q}{P} .
$$

It can be easily implied form Eq. (7) that for $\varphi=0$ no reactive power is produced $(P=S)$. It has to be emphasized that the power factor $(P F)$ equals to $\cos \varphi$ when there exists only the fundamental harmonic of current or if higher harmonics are neglectable. Otherwise, one has to use the following formula:

$$
P F=\frac{1}{\sqrt{1+\mathrm{THD}_{I}^{2}}} \cos \varphi=\frac{1}{\sqrt{1+\left(\frac{I_{1, \mathrm{RMS}}}{I_{\mathrm{RMS}}}\right)^{2}}} \cos \varphi,
$$

where $I_{\mathrm{RMS}}$ is the total current and $I_{1, \mathrm{RMS}}$ denotes the fundamental component of current. RMS subscript means that both are computed as root mean squarevalues.

For our further considerations we choose $\tan \varphi$ as more handy for expressing the phase shift. It is due to a $\operatorname{sign}$ of the $\tan \varphi$ function which reflects a type of reactive power. A phase shift can be either negative or positive. In the former case reactive power is referred to as a capacitive and in the latter - as an inductive one.

In the case of deformed waveforms $[4,23]$, in order to correctly determine the values of reactive power, the definition of reactive power according to Budeanu's theory has to be used, then:

$$
Q_{B}=\sum_{h=1}^{\infty} u_{h} i_{h} \sin \varphi_{h}
$$

where the sum iterates over all current and voltage harmonics.

In the considered case we deal with dimmed LED fixtures for which dimming implies changes of the current components $\left\{i_{h}\right\}$ only while the voltage remains 
unchanged, i.e., all harmonics except the fundamental are 0 : $\left(u_{h} \approx 0\right.$ for $\left.h>1\right)$. Thus Eq. (9) reduces to the fundamental component only: $Q_{B}=u_{1} i_{1} \sin \varphi_{1}$.

We focus on the compensation model in which we try to fit with $\tan \varphi$ within the range of $\left[0, \tan \varphi_{0}\right]$. CRP compensation $\left(Q_{c a p}<0\right)$ is achieved by increasing inductive reactive power $\left(Q_{\text {ind }}>0\right)$ so that a resultant $\tan \varphi$ is at least nonnegative and it is less than $\tan \varphi_{0}$. It can be written by means of Eq. (7):

$$
\tan \varphi=\frac{Q_{c a p}+Q_{\text {ind }}}{P}
$$

In our analysis we consider $Q_{\text {cap }}=Q_{\text {fix }}+Q_{\text {power line }}$ being a sum of two negative components: the first component corresponds to a LED fixture and the second one is associated with a power line which acts as a capacitor. Although for short distances it is neglectable, for longer ones, however, it can yield capacity which has to be taken into account to avoid a further inaccurate compensation.

For a fixture working with some established dimming level and its power line, a fixed source of the inductive reactive power, $Q_{i n d}$, compensating system with a constant reactance is employed. A reactance, $X_{C}$, of a fixture and its power line can be calculated according to the formula:

$$
X_{C}=\frac{1}{\frac{\left|Q_{\mathrm{fix}}\right|}{U^{2}}+\omega c l},
$$

where $U=230 \mathrm{~V}$ (in Europe) is a voltage value, $\omega=2 \pi f$ (the frequency $f=$ $50 \mathrm{~Hz}$ in Europe), $Q_{\text {fix }}$ stands for a CRP of a fixture, $c$ denotes a cable capacity per unit length and $l$ is a cable length. From the practical point of view, the capacitive reactive power compensation can be most easily achieved by attaching a parallel choke with an inductive reactance $X_{L}$ to a fixture, such that $X_{L}=X_{C}$. Since an inductive reactance is given by the formula:

$$
L=\frac{X_{L}}{\omega}
$$

where $L$ is an inductance, one can obtain the desired value of the latter by combining Eqs. (11), (12) and assuming equality of reactances $X_{C}$ and $X_{L}$ :

$$
L=\frac{1}{\frac{\omega\left|Q_{\mathrm{fix}}\right|}{U^{2}}+\omega^{2} c l} .
$$

It should be remarked that such an approach allows to transform the highly penalized CRP into the inductive one with an acceptable $\tan \varphi<\tan \varphi_{0}$.

\section{Single Luminaire Example}

As a test case we have examined an Ampera Midi $106 \mathrm{~W}$ manufactured by Schréder. It is controlled using a $0-10 \mathrm{~V}$ dimmer. For the control signal $s$ in the range $7.5-10 \mathrm{~V}$ the active power $P$ is practically constant. In turn, $P$ becomes 
linearly depending on $s$ when $s \in[0.5 \mathrm{~V}, 7.5 \mathrm{~V}]$. This behavior can be seen in Fig. 1. Since the fixture's luminous flux intensity $\lambda$ is proportional to the active power $\left(\lambda \approx \frac{P}{P_{\max }} \times 100 \%\right)$ being proportional to the control signal, if $s \leq 7.5 \mathrm{~V}$, we can identify the control signal voltage with $\lambda$ when the former is within the range $0-7.5 \mathrm{~V}$.

Hereafter, every time when we talk about "setting/adjusting $\lambda$ " we mean such setting of $s$ so that a desired value of $\lambda(s)$ is obtained.

The Ampera Midi $106 \mathrm{~W}$ fixture measurements are given in Table 1. Besides the active power and $\cos \varphi$, calculated values of $\tan \varphi$ are also shown. Their negative sign is implied by the capacitive nature of the reactive power for which $\varphi<0$.

Table 1. The characteristics of the Ampera Midi $106 \mathrm{~W}$ fixture, gathered remotely for a series of control signals, $P$ denotes an active power

\begin{tabular}{|l|l|l|l|l|l|l|l|l|l|l|l|l|l|l|l|l|l|l|l|l|}
\hline $\mathrm{Ctrl}[\mathrm{V}]$ & 0.5 & 1.0 & 1.5 & 2.0 & 2.5 & 3.0 & 3.5 & 4.0 & 4.5 & 5.0 & 5.5 & 6.0 & 6.5 & 7.0 & 7.5 & 8.0 & 8.5 & 9.0 & 9.5 & 10 \\
\hline \hline$P[\mathrm{~W}]$ & 20,8 & 26,3 & 31,9 & 39,4 & 44,9 & 52,5 & 58,3 & 65,7 & 71,8 & 79,9 & 85,9 & 91,8 & 97,5 & 105,9 & 111,7 & 111,6 & 110,7 & 111,4 & 111,1 & 111,1 \\
\hline $\cos \varphi$ & 0,52 & 0,59 & 0,69 & 0,76 & 0,80 & 0,84 & 0,85 & 0,88 & 0,89 & 0,90 & 0,91 & 0,92 & 0,92 & 0,93 & 0.94 & 0.94 & 0.94 & 0.94 & 0.94 & 0.94 \\
\hline $\tan \varphi$ & $-1,64$ & $-1,37$ & $-1,05$ & $-0,86$ & $-0,75$ & $-0,65$ & $-0,62$ & $-0,54$ & $-0,51$ & $-0,48$ & $-0,46$ & $-0,43$ & $-0,43$ & $-0,40$ & -0.36 & -0.36 & -0.36 & -0.36 & -0.36 & -0.36 \\
\hline
\end{tabular}

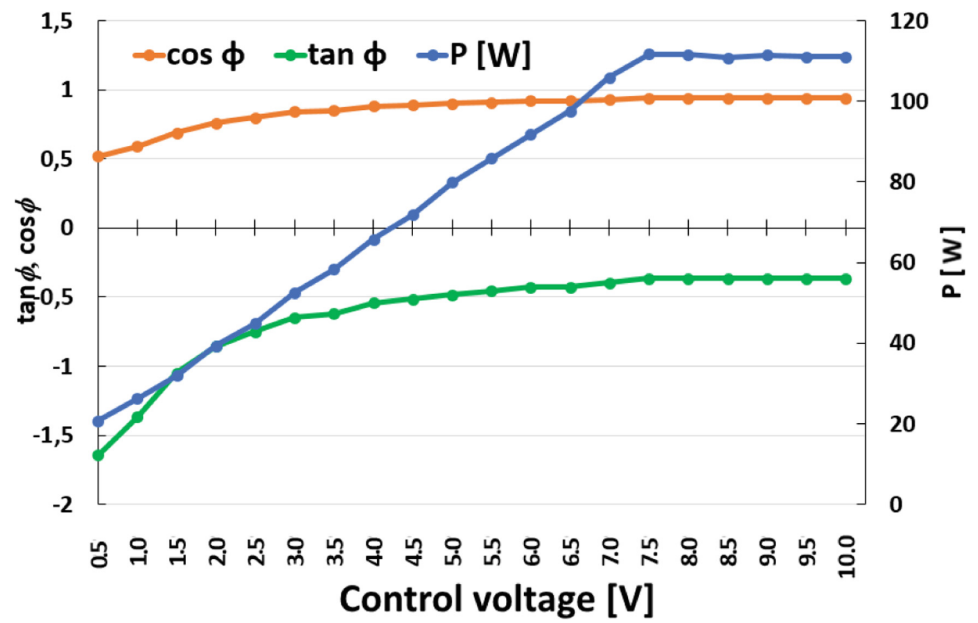

Fig. 1. The performance chart of the Ampera Midi $106 \mathrm{~W}$ fixture for data presented in Table 1

Having the above data we find the minimum and maximum values of the fixture's CRP $Q_{\text {fix }}(\lambda)=P(\lambda) \tan (\lambda): Q_{\text {fix }}^{\min }=-41.85$ Var and $Q_{\text {fix }}^{\max }=-33.46$ Var, reached for $\lambda_{1}$ and $\lambda_{2}$ respectively. The capacitive reactive power component contributed by the power line is constant. To correct a CRP of the fixture a 
choke generating an inductive reactive power $Q_{i n d}=-Q_{\text {fix }}^{\min }$ has to be used. Thus we get

$$
X_{L}=\frac{U^{2}}{Q_{\text {ind }}} .
$$

After adding the above inductance to the circuit the vales $\tan \varphi\left(\lambda_{1}\right)$ and $\tan \varphi\left(\lambda_{2}\right)$ change. From the Eq. (10) we have (hereafter, a primed $\varphi$ will refer to a phase shift after compensation) $\tan \varphi\left(\lambda_{1}\right) \rightarrow \tan \varphi^{\prime}\left(\lambda_{1}\right)=0$ and

$$
\tan \varphi\left(\lambda_{2}\right) \rightarrow \tan \varphi^{\prime}\left(\lambda_{2}\right)=\frac{Q_{c a p}\left(\lambda_{2}\right)+Q_{i n d}}{P\left(\lambda_{2}\right)}=0.37
$$

For this case $\tan \varphi^{\prime}\left(\lambda_{2}\right)<\tan \varphi_{0}$. Otherwise (the case referred to as overcompensation), lamp should not be dimmed to the values which violate this constraint unless a business analysis points that paying given charges for overcompensation is still more profitable.

\section{Capacitive Reactive Power Compensation in Large Lighting Installations}

In this section we begin with the basic case of compensation, made for a single control cabinet. Next we proceed to the case of multiple cabinets for which the issue of high analytical complexity of the system arises.

\subsection{Case 1. Control Cabinet}

Compensation for a series of lamps connected to a control cabinet is made similarly as in the above example. We compensate the total CRP being a sum over all fixtures: $\mathbf{Q}_{\text {cap }}=\sum_{i=1}^{n}\left(Q_{\mathrm{fix}, i}+Q_{\text {power line,i }}\right)$, where $Q_{\mathrm{fix}, i}=Q_{\mathrm{fix}, i}\left(\lambda_{i}\right)$ is a $\mathrm{CRP}$ of $i$-th fixture (depending on its luminous flux ratio $\lambda_{i}$ ) and $Q_{\text {power line,i }}$ is a CRP contributed by the corresponding power line.

Getting the extreme values of $\mathbf{Q}_{c a p}$ is not straightforward, however, as the fixtures are not assumed to be identical, dimmed equally and in the same time moments. It should be noted that for a dynamically controlled lighting system both minimum and maximum, $\mathbf{Q}_{c a p}^{\min }, \mathbf{Q}_{c a p}^{\max }$, are calculated over some time period, e.g., operating hours. Namely, with such assumptions $\lambda_{i}=\lambda_{i}(t)$ is a function of time and $\mathbf{Q}_{\text {cap }}(t)=\mathbf{Q}_{\text {cap }}\left(\lambda_{1}, \lambda_{2}, \ldots, \lambda_{n}\right)$ so is. In practice both the time variable and $\lambda_{i}$ 's are discretized so

$$
\mathbf{Q}_{c a p}^{\min }=\min _{t} \mathbf{Q}_{c a p}(t), \quad \mathbf{Q}_{c a p}^{\max }=\max _{t} \mathbf{Q}_{c a p}(t)
$$

can be calculated programatically.

It should be remarked that time discretization is possible because changes of $\lambda$ 's are triggered by varying environment conditions being evaluated as time averages (for example, an increased average traffic flow). $\lambda$ adjustments are always made in compliance with mandatory lighting standards [7]. 
That step is important because due to functional specificity of particular lamps and the control patterns usually $\mathbf{Q}_{c a p}^{\min } \neq \sum_{i=1}^{n}\left(Q_{\mathrm{fix}, i}^{\min }+Q_{\text {power line, } \mathrm{i}}\right)$ which means that usually minima of the CRP of particular lamps do not meet in the one moment. For this reason $\mathbf{Q}_{c a p}^{\min }$ (but also $\mathbf{Q}_{c a p}^{\max }$ ) cannot be easily assessed.

The value of inductance $L$ is derived starting from the minimum CRP reachable by the installation, i.e., $\mathbf{Q}_{c a p}^{\mathrm{min}}$. We set a required value of the inductive reactive power to be $Q_{i n d}=-\mathbf{Q}_{c a p}^{\min }$. Having that one obtains $L$ using (12) and (14): $L=U^{2} / \omega Q_{\text {ind }}$.

In the case of several circuits connected to a cabinet (e.g., for a 3-phase power line) each of them is considered separately. In particular each circuit will be attached with a dedicated compensator.

\subsection{Case 2. Multiple Cabinets}

The scheme presented in the previous subsection applies also to multiple cabinets (for brevity referred to as a lighting grid) which are compensated separately as discussed above. If a control cabinet has several circuits then we can divide it logically, for computational purposes, into virtual, single-circuit cabinets.

The difference between a single cabinet and a lighting grid is significantly higher complexity of the latter. This complexity refers to the lighting infrastructure topology and functional relations as seen from the perspective of control (adjusting $\lambda$ 's for particular fixtures) in terms of relations among control cabinets, circuits, lamps and streets segments.

A value of $\tan \varphi_{G}$ for a non-corrected lighting grid is computed as:

$$
\tan \varphi_{G}\left(\lambda_{1}, \lambda_{2}, \ldots \lambda_{n}\right)=\frac{Q^{*}+\sum_{i} Q_{\mathrm{fix}, i}\left(\lambda_{i}\right)}{\sum_{i} P_{i}\left(\lambda_{i}\right)},
$$

where $Q^{*}$ is a total CRP of power lines and both sums iterate over all fixtures. As previously, $\lambda_{i}$ 's are time dependent. The general compensation scheme remains unchanged, i.e., we correct $\tan \varphi_{G}$ by adding such inductive reactive powers to particular circuits that their total value is $Q_{i n d}=-\left(Q^{*}+\min _{t} \sum_{i} Q_{\mathrm{fix}, i}\right)$, where $\min _{t}$ denotes a minimum over the time. Thus, new $\tan \varphi_{G}$ is given as:

$$
\tan \varphi_{G}^{\prime}\left(\lambda_{1}, \lambda_{2}, \ldots, \lambda_{n}\right)=\frac{Q_{\text {ind }}+Q^{*}+\sum_{i} Q_{\mathrm{fix}, i}\left(\lambda_{i}\right)}{\sum_{i} P_{i}\left(\lambda_{i}\right)} .
$$

The major difficulty for the case of multiple cabinets is finding an optimal configuration of compensators in a reasonable time rather than a compensation as such, particularly for dynamically controlled lighting systems. An alternative to the static compensation could be using a dynamic VAR compensator. It generates, however, significantly higher cost, especially when multiplied by the number of cabinets. For this reason we focus on building an effective computational approach which enables planning configuration of compensators for a large lighting grid. 


\section{Algorithmic Approach to Compensation Planning}

In this section we address problem of compensation planning by introducing the formal representation of a lighting installation and the algorithm which finds optimal configuration of static compensators.

\subsection{Problem Overview}

The luminaires illuminating public spaces (grouped into sets denoted by $\mathcal{L}$ in Fig. 2), also referred to as the light points, are organized in the hierarchical manner. At the lowest level we have single light points which are grouped in circuits. One or more circuits, dependently on a local specificity, are connected to a control cabinet (denoted by $C$ ).

A roadway network is assumed to be logically divided into the segments (denoted by $S$ in Fig. 2). The partition is made in such a way that a given segment is a uniform street geometry assigned with a specific lighting class depending on such factors as a function of a relevant area (parking zone, road junction, freeway, residential area etc.), traffic flow and others $[2,7,11,14]$. The important fact related to the lighting class assignment is that standards admit changing a class if local conditions change, e.g, when the traffic flow level increases (then some more restrictive class, implying higher luminance, can be selected) or decreases (the class resulting in lower luminance required for this area is assigned). Changing an actual class allows decreasing luminous fluxes of relevant fixtures (i.e., decreasing corresponding $\lambda$ 's, dimming them) and thus obtain energy savings.

Our goal is to set such compensators in a lighting grid so that (see Eq. (16)): $0 \leq \tan \varphi_{G}^{\prime}\left(\lambda_{1}, \lambda_{2}, \ldots, \lambda_{n}\right)<\tan \varphi_{0}$. This task is not straightforward because the following factors have to be taken into account:

$-\lambda$ 's of all relevant fixtures. Note that a value of $\lambda$ depends on a fixture model but also on a public area (segment) being illuminated: a fixture's luminous flux in a residential area is usually lower than in a road junction zone. For this reason two luminaires equipped with the same fixture model can be set to two different $\lambda$ 's and thus produce two different capacitive reactive powers. It implies that we cannot simply multiply a number of fixtures of a given type by a unit reactive power, when computing $\sum_{i} Q_{\mathrm{fix}, i}$.

- An $i$-th fixture, $f_{i}$, installed by a segment $S$ can be dimmed due to the lighting class reduction so one deals with the time-dependent (and, in the general case, unpredictable) profiles of the active and reactive power of $f_{i}$ : $P_{i}(t), Q_{\mathrm{fix}, i}(t)$ (for the simplicity we can assume that $t$ changes in a 24 -h period). Obviously, the same applies to other fixtures illuminating $S$ and to the remaining segments. Thus we have to analyze the time coincidences of the particular power profiles. Note that it may easily happen that the simultaneous dimming of all fixtures never occurs: the maxima and minima of the real and reactive powers never overlap so we are unable to make $a$ priori any rough estimation of $\tan \varphi_{G}^{\prime}$. 
Having in mind the above one has to find $\tan \varphi_{G}^{\prime}$ iterating over entire lighting installation covering all cabinets and segments, and over its all accessible states (dimming levels). This task cannot be achieved "manually" for its high computational complexity and the computer aided solution finding has to be applied. Prior to this, however, we need to build a formal data model to express the problem in terms applicable for a computer system. The following subsections contain both problem formalization and the compensation algorithm.

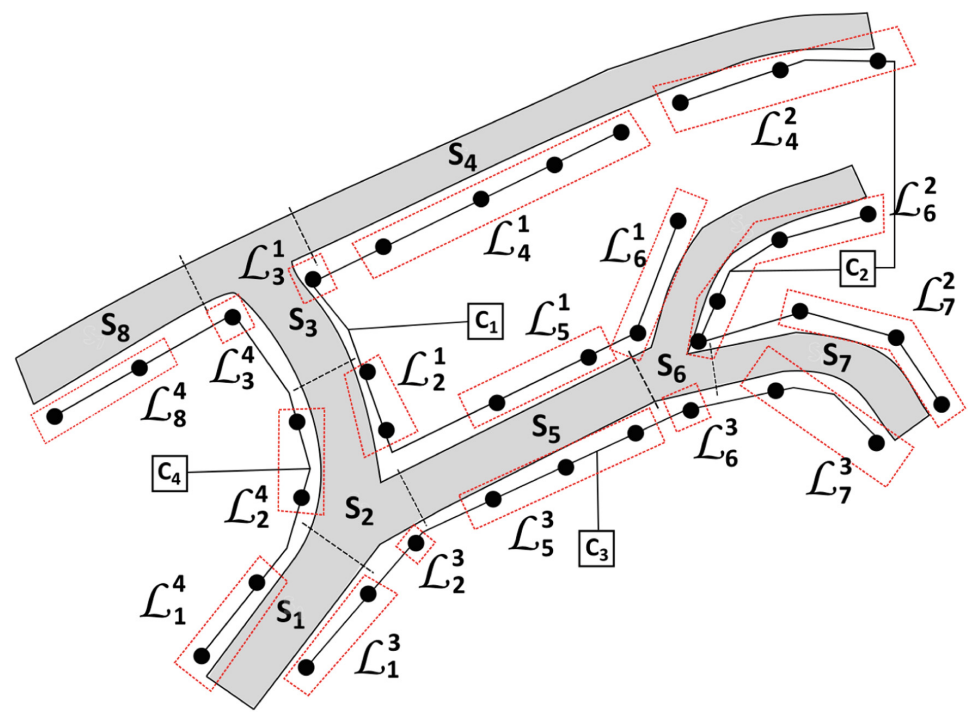

Fig. 2. The sample road and installation layout. $S_{1}, \ldots, S_{8}$ denote road segments (delimited by dashed lines); $C_{1}, \ldots, C_{4}$ are control cabinets; bold dots denote particular luminaires; $\mathcal{L}_{i}^{k}$ is a list of lamps (bounded by red dotted lines): a lower index (i) refers to a relevant segment number; an upper $(k)$ stands for a number of cabinet to which luminaires from $\mathcal{L}_{i}^{k}$ are attached. (Color figure online)

\subsection{Formal Representation}

As mentioned above, we divide a system of roadways, walkways and squares being illuminated into segments $S_{1}, S_{2}, \ldots S_{m}$ such that in a given moment entire segment $S_{i}$ is assigned with a single lighting class. Additionally it is assumed that we consider only the segments being illuminated by at least one luminaire. A subset of luminaires powered by $C_{k}(k=1, \ldots, n)$ and illuminating a segment $S_{i}$ will be denoted as $\mathcal{L}_{i}^{k}$. It should be noted that in general case it can occur that $\mathcal{L}_{i}^{k} \cap \mathcal{L}_{i}^{k^{\prime}} \neq \emptyset$ for $k \neq k^{\prime}$ what means that some luminaires can illuminate more than one segment. It can be easily checked that $\sum_{k}\left|\mathcal{L}_{i}^{k}\right|$ is a number of all luminaires illuminating an $i$-th segment. In turn, $\left|\bigcup_{i} \mathcal{L}_{i}^{k}\right|$ is a total number of luminaires attached to a $k$-th cabinet. 
To enclose the above model within a single structure passed to an algorithm we define the notion of a structure matrix:

$$
\mathbf{M}=\left\{\mathcal{L}_{i}^{k}\right\}_{i=1,2, \ldots, m}^{k=1,2, \ldots, n},
$$

where $n$ and $m$ are numbers of cabinets and segments respectively. If no luminaire powered by $C_{k}$ illuminates $S_{i}$ then we denote $\mathcal{L}_{i}^{k}=\emptyset$.

For the sample layout shown in Fig. 2 we have the matrix $\mathbf{M}$ which aggregated form is shown in Table 2: particular lists, $\mathcal{L}_{i}^{k}$, are replaced by their lengths (numbers of contained luminaires).

Table 2. Aggregated matrix representation of the layout shown in Fig. 2. For better readability all cells for which $\left|\mathcal{L}_{i}^{k}\right|=0$ are left blank.

\begin{tabular}{l|l|l|l|l|l|l|l|l|l}
\hline \multicolumn{2}{c|}{} & \multicolumn{9}{|l}{ Segment } \\
\cline { 3 - 11 } \multicolumn{1}{c|}{} & $S_{1}$ & $S_{2}$ & $S_{3}$ & $S_{4}$ & $S_{5}$ & $S_{6}$ & $S_{7}$ & $S_{8}$ \\
\hline \multirow{3}{*}{ Cabinet } & $C_{1}$ & & 2 & 1 & 4 & 2 & 2 & & \\
\cline { 2 - 10 } & $C_{2}$ & & & & 3 & & 4 & 3 & \\
\cline { 2 - 10 } & $C_{3}$ & 2 & 1 & & & 3 & 1 & 2 & \\
\cline { 2 - 10 } & $C_{4}$ & 2 & 2 & 1 & & & & & 2 \\
\hline
\end{tabular}

\subsection{Compensation Strategy - Algorithmic Approach}

The goal of the algorithm (see Algorithm 1) is to determine a list of compensators' inductances such as a sum (denoted as $\Delta$ ) of charges corresponding to exceeding the $\tan \varphi_{0}$ threshold and the annual power usage expenses, is minimized. Optionally, an additional constraint may be imposed on a total cost of compensators (being a onetime outlay).

As an algorithm's input we put a structure matrix (17) representing links between control cabinets and illuminated segments. Note that elements of $\mathbf{M}$ contain all relevant data required in further processing. An output is a sequence of inductances $\left\{L_{k}\right\}$ for subsequent cabinets.

Several comments related to the algorithm (see Algorithm 1) performance should be given.

1. The pseudocode has two nested loops (the first in the line 4 and the second, implicit, triggered in the line 11), except the finite iteration over cabinets (line $5)$. Both loops are protected against an infinite execution so stop condition is satisfied for each algorithm run.

2. A source of the computational complexity of the presented algorithm are operations of finding minimum and maximum, located in lines 6 and 8 respectively. To reduce the number of operations one can apply the problem-specific heuristics. 


\section{Algorithm: GetCompensators $(\mathbf{M})$}

Input : $\mathbf{M}$ - structure matrix

Output: $\left\{L_{k}\right\}$-inductances of compensators

2 begin

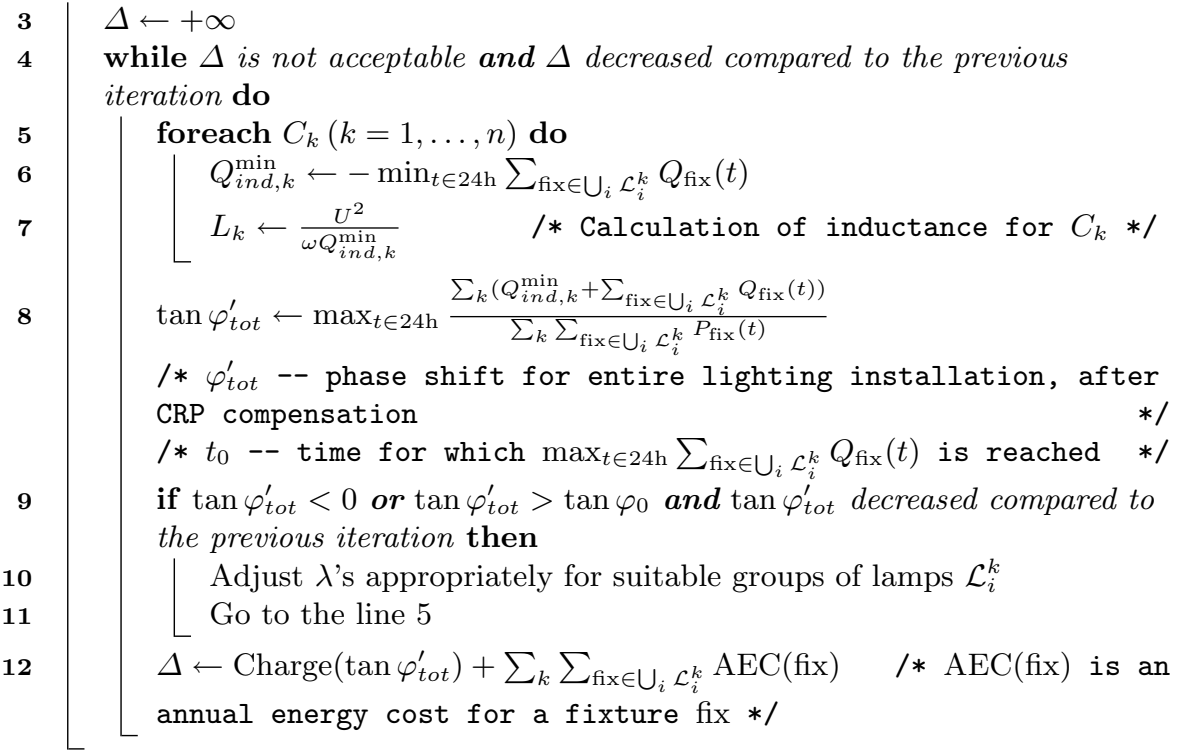

Algorithm 1: Calculating inductances of multiple compensators.

3. The function Charge $(\tan \varphi)$ used in the line 12 represents annual (or monthly, depending on needs) due charges related to exceeding $\tan \varphi_{0}$. Thus $\Delta$ computed in the same line stands for the accumulated annual costs of roadway illumination and potential fees for reactive power generation. The premises for evaluating the condition contained in the line 4 ( $\Delta$ is not acceptable) have a business character. It is based on priorities defined by a lighting infrastructure owner or operator.

4. The step given in the line 10 may look imprecise so its clarification is needed. As pointed in the previous sections, the $\lambda$ parameters for particular luminaires are responsible for changes of $\tan \varphi$. Thus, in the case of exceeding the $\tan \varphi_{0}$ limit we aim at changing $\lambda$ 's for relevant fixtures. Their "relevance" can be stated only on the basis of a known, specific installation's layout. The restriction which can be made here is focusing on luminaires from a set $\mathcal{L}_{i}^{k}$ of lamps illuminating given segment(s) $S_{i}$ and/or connected to given cabinet(s) $C_{k}$. Their selection is also layout-specific.

5. The very important issue which has to be addressed in CRP compensation planning for lighting systems are failures of fixtures. They cause increasing of $Q_{c a p}$ in the numerator of Eq. (10) and thus can lead to significant growth of $\tan \varphi$ value and over-compensation. Basing on statistics and historical data, 
a lighting system operator should put a buffer, in terms of $\lambda$ values (see the line 10 of the algorithm), to protect the system against such events.

\section{Conclusions}

Thanks to the presented algorithm we are able to achieve the low-cost static compensation of capacitive reactive power generated in LED-based lighting systems. This approach is proposed as an alternative to dynamic VAR compensation being significantly more expensive. The price ratio between the dynamic and static method, for $k$ circuits connected to a single cabinet is of the order of $10: k$ (the Polish market as of 2019). Note that it is an estimated value only, depending on multiple factors such as a control cabinet load or a power line type but also equipment order volume or a negotiated manufacturer's offer.

If fitting under the $\tan \varphi_{0}$ threshold is not feasible for the entire installation, e.g., due to the system dynamics, the control patterns, $\lambda_{i}(t)$ should be adjusted to meet this constraint. An alternative approach is finding a trade-off between costs related to the inductive reactive power generation and the savings obtained by improving energy efficiency of an installation.

The presented calculations are possible for lighting standard [7] compliant installations. It implies having all luminaire parameters, such as power and dimming levels for particular lighting classes calculated, and information about their geographical distribution and actual electric connections accessible.

\section{References}

1. Asian Development Bank: LED Street Lighting Best Practices (2017). https://goo. gl/i75Ztk. Accessed 21 Jan 2018

2. Australian/New Zealand Standard: AS/NZS 1158.1.1:2005 Lighting for roads and public spaces Vehicular traffic (Category V) lighting - Performance and design requirements. SAI Global Limited (2005)

3. Cheng, C.A., Chang, C.H., Cheng, H.L., Chang, M.T.: A novel LED driver with power factor correction suitable for streetlight applications. In: 2017 IEEE 3rd International Future Energy Electronics Conference and ECCE Asia (IFEEC 2017 - ECCE Asia), pp. 1220-1223, June 2017

4. Czarnecki, L.S.: Considerations on the reactive power in nonsinusoidal situations. IEEE Trans. Instrum. Meas. IM-34(3), 399-404 (1985). https://doi.org/10.1109/ tim.1985.4315358

5. Dazzletek: Dazzletek Lighting Control System (2015). http://goo.gl/ZcJAvL. Accessed 3 Mar 2015

6. Ekrias, A., Eloholma, M., Halonen, L.: An advanced approach to road lighting design, measurements and calculations. Technical Report (53) (2008). http://goo. $\mathrm{gl} / 3 \mathrm{IVBnc}$

7. European Committee For Standarization: Road Lighting. Performance requirements, EN 13201-2:2015 (2015)

8. Federal Energy Regulatory Commission: Principles for Efficient and Reliable Reactive Power Supply and Consumption, Staff Report, Docket No. AD05-1-000, 4 February 2005 
9. Gómez-Lorente, D., Rabaza, O., Estrella, A.E., Peña-García, A.: A new methodology for calculating roadway lighting design based on a multi-objective evolutionary algorithm. Expert Syst. Appl. 40(6), 2156-2164 (2013). http://goo.gl/bx4cqn

10. Han, J.H., Lim, Y.C.: Design of an LLC resonant converter for driving multiple LED lights using current balancing of capacitor and transformer. Energies 8(3), 2125-2144 (2015). http://www.mdpi.com/1996-1073/8/3/2125

11. Illuminating Engineering Society of North America (IESNA): American National Standard Practice For Roadway Lighting, RP-8-14. IESNA, New York (2014)

12. Kostic, M., Djokic, L.: Recommendations for energy efficient and visually acceptable street lighting. Energy 34(10), 1565-1572 (2009). http://goo.gl/KQ64v6. 11th Conference on Process Integration, Modelling and Optimisation for Energy Saving and Pollution Reduction

13. Lee, E.S., Choi, B.H., Nguyen, D.T., Choi, B.G., Rim, C.T.: Long-lasting and highly efficient TRIAC dimming LED driver with a variable switched capacitor. J. Power Electron. 16, 1268-1276 (2016)

14. de l'Eclairage, C.I.: Lighting of roads for motor and pedestrian traffic, CIE 115:2010. CIE, Vienna (2010)

15. Minister of Economy of Poland: Rozporządzenie Ministra Gospodarki z dnia 18 sierpnia $2011 \mathrm{r}$. w sprawie szczegółowych zasad kształtowania i kalkulacji taryf oraz rozliczeń w obrocie energią elektryczną (2011). http://prawo.sejm.gov.pl/isap.nsf/ download.xsp/WDU20111891126/O/D20111126.pdf. Dz.U. 2011 nr 189 poz. 1126

16. Molina-Moreno, V., Leyva-Díaz, J.C., Sánchez-Molina, J., Peña García, A.: Proposal to foster sustainability through circular economy-based engineering: a profitable chain from waste management to tunnel lighting. Sustainability $\mathbf{9}(12)$ (2017). https://doi.org/10.3390/su9122229. http://www.mdpi.com/2071-1050/9/12/2229

17. Peña-García, A., Gil-Martín, L., Hernández-Montes, E.: Use of sunlight in road tunnels: an approach to the improvement of light-pipes' efficacy through heliostats. Tunn. Undergr. Space Technol. 60, 135-140 (2016). https://doi.org/10. 1016/j.tust.2016.08.008. http://www.sciencedirect.com/science/article/pii/S08867 79815302121

18. Philips: CityTouch (2015). http://goo.gl/GhCJsX. Accessed 2 Mar 2015

19. Salata, F., et al.: Energy optimization of road tunnel lighting systems. Sustainability $7(7), 9664$ (2015). https://doi.org/10.3390/su7079664. http://www.mdpi.com/ 2071-1050/7/7/9664

20. Sędziwy, A.: A new approach to street lighting design. LEUKOS 12(3), 151-162 (2016)

21. Sędziwy, A., Basiura, A.: Energy reduction in roadway lighting achieved with novel design approach and LEDs. LEUKOS 14(1), 45-51 (2018)

22. Schréder: Owlet (2015). http://goo.gl/9g53OP. Accessed 2 Mar 2015

23. Vieira, D., Shayani, R.A., de Oliveira, M.A.G.: Reactive power billing under nonsinusoidal conditions for low-voltage systems. IEEE Trans. Instrum. Meas. 66(8), 2004-2011 (2017). https://doi.org/10.1109/tim.2017.2673058

24. Wojnicki, I., Kotulski, L.: Street lighting control, energy consumption optimization. In: Rutkowski, L., Korytkowski, M., Scherer, R., Tadeusiewicz, R., Zadeh, L.A., Zurada, J.M. (eds.) ICAISC 2017. LNCS (LNAI), vol. 10246, pp. 357-364. Springer, Cham (2017). https://doi.org/10.1007/978-3-319-59060-8_32

25. Worcester Energy: Street Lighting Retrofit Project. Progress Report (2017). https://goo.gl/pktcCp. Accessed 21 Jan 2018 